\title{
Contrast sensitivity to angular frequency gratings is not higher than to Cartesian gratings
}

Y. Zana and

A.C.G.T. Cavalcanti
Departamento de Psicologia, LabVis, CFCH,

Universidade Federal de Pernambuco, Recife, PE, Brasil

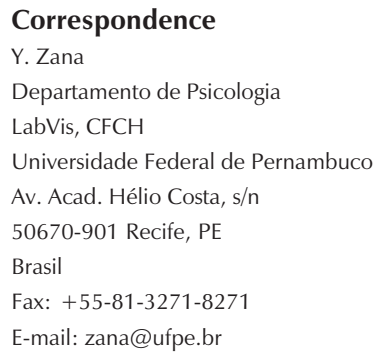

Received August 20, 2002 Accepted September 5, 2003

\begin{abstract}
When contrast sensitivity functions to Cartesian and angular gratings were compared in previous studies the peak sensitivity to angular stimuli was reported to be $0.21 \log$ units higher. In experiments carried out to repeat this result, we used the same two-alternative forcedchoice paradigm, but improved experimental control and precision by increasing contrast resolution from 8 to 12 bits, increasing the screen refresh rate from $30 \mathrm{~Hz}$ interlaced to $85 \mathrm{~Hz}$ non-interlaced, linearizing the voltage-luminance relation, modulating luminance in frequencies that minimize pixel aliasing, and improving control of the subject's exposure to the stimuli. The contrast sensitivity functions to Cartesian and angular gratings were similar in form and peak sensitivity (2.4 cycles per visual degree (c/deg) and $32 \mathrm{c} / 360^{\circ}$, respectively) to those reported in a previous study ( $3 \mathrm{c} / \mathrm{deg}$ and $32 \mathrm{c} / 360^{\circ}$, respectively), but peak sensitivity to angular stimuli was $0.13 \log$ units lower than that to Cartesian stimuli. When the experiment was repeated, this time simulating the experimental control level used in the previous study, no difference between the peak sensitivity to Cartesian and angular stimuli was found. This result agrees with most current models that assume Cartesian filtering at the first visual processing stage. The discrepancy in the results is explained in part by differences in the degree of experimental control.
\end{abstract}

\section{Introduction}

In 1968 Campbell and Robson (1) suggested that the human visual system has groups of independent filters sensitive to a narrow band of Cartesian frequencies and that the contrast sensitivity function (CSF) corresponds to the sensitivity envelope of all these channels. According to this hypothesis, the visual system executes an analysis or a decomposition of an image into its elementary components, a kind of Fourier analysis of the retinal image (2). Accordingly, detec-
Key words

- Contrast sensitivity functions

coordinates

- Radial and angular gratings
- Cartesian and polar tion threshold is determined by the Fourier amplitude of the spatial components, and not by the total contrast of the image.

Stimuli defined in polar coordinates were suggested by Kelly (3) to probe the human visual system. We adopted the terms Cartesian, radial and angular stimuli for gratings whose luminance is modulated along the horizontal and/or vertical axes, the radius of a circle, and the angle within a circle, respectively. These terms were also used when describing stimuli used by others under different names. These stimuli would be sym- 
metrically circular and their luminance would vary as a function of the cylindrical J0 Bessel function. Kelly thought that these stimuli would be more "natural" and provide a fixation center that Cartesian gratings do not offer. It was argued, on mathematical grounds, that the contrast sensitivity to this kind of stimuli would not be different from that to sine gratings. Later, Kelly and Magnuski (4) compared the CSFs to Cartesian and radial J0 grating stimuli. Surprisingly, it was found that the sensitivity to Cartesian gratings was higher than that for radial J0 (except at frequencies below one cycle per visual degree, c/deg). It was speculated that the differences in the CSFs could be explained by the circular symmetry or the local contrast variations, both present in the radial J0 stimuli. To identify which of the two factors is responsible for the results, the CSF of radial cosine stimuli was measured. This kind of stimulus was selected because it is circularly symmetric like the $\mathrm{J} 0$ stimuli but lacks the local contrast variations. If its CSFs were equal to that of the $\mathrm{J} 0$ gratings, it could be concluded that the circular symmetry is responsible for the differences from the Cartesian gratings. If, on the other end, the CSFs were equal to that of the Cartesian gratings, it could be concluded that the local contrast variations are responsible for the differences in CSFs between the radial J0 and Cartesian stimuli. The result was an intermediate difference and it was concluded that the circular symmetry, as well as the local contrast variations, are responsible for the differences. To explain these results, the stimuli were analyzed into their Fourier spectral components. It was shown that the maximum amplitude Fourier component of radial J0 gratings varied inversely with the frequency while that of the Cartesian gratings also varied, but to a lesser extent. The relation between the radial $\mathrm{J} 0$ and Cartesian grating maximum amplitude Fourier component varied as a function of the 1 st power, while that of the cosine gratings varied as a function of the 0.5 power. Similar relations were found between the CSF of the radial J0 or the radial cosine stimuli and the Cartesian gratings. The authors concluded that the detection threshold of radial gratings could be explained by a peak detection model.

In 1990 Simas and Dodwell (5) suggested the existence of spatial filters, whose elementary stimuli would be defined in angular frequencies, and that are orthogonal to presumed radial Bessel filters. Simas and colleagues evaluated this hypothesis in a series of studies (5-9). They measured the CSF to stimuli defined as angular coordinates, such that luminance varied sinusoidally with angle within a circle $(5,8)$ and found that the CSF had approximately one $\log$ unit gain and plateau peak sensitivity between 13 and 32 cycles. In the second study (8) they also compared the result with the CSF to Cartesian gratings. Interestingly, the peak sensitivity was approximately 0.21 $\log$ units lower than that for angular gratings. However, this result is not predicted by the model that assumes that detection threshold is explained by the peak Fourier amplitude of the image, since in angular stimuli the total contrast energy is divided between Fourier components of different frequencies and orientations, while in Cartesian stimuli the contrast energy is concentrated in a single Fourier component.

Due to the importance of the issue and the fact that this result has never been reported to have been reproduced by other investigators, we proposed to replicate their experimental design, but with several control improvements, as will be discussed below. The results indicate that contrast sensitivity to angular stimuli is lower than that to Cartesian stimuli, as predicted by the peak detection model.

\section{Material and Methods}

\section{Subjects and equipment}

The two authors participated in the ex- 
periments. Both were corrected myopes and practiced prior to data collection. The stimuli were generated on a color monitor by a graphics board used at a resolution of $1024 \mathrm{x}$ 768 pixels at a frame rate of $85 \mathrm{~Hz}$ noninterlaced. The graphics board had $256 \mathrm{lu}-$ minance level for each channel. To increase the number of luminance levels available to 4096, the red and blue color channels of the graphics board were attenuated and combined in a resistance network (10). The combined monochrome signal was connected to the green input of the monitor to produce a luminance-modulated image. An OptiCAL photometer (Cambridge Research Systems, Rochester, Kent, England) was used to measure the voltage-luminance function, from which the 4096-level look-up table was constructed. The final linear correlation of the screen luminance was 0.9998. Luminance measurements in the stimuli area guaranteed that spatial non-linearity was below $4 \%$.

\section{Stimulus definition}

CSFs were measured for the same two stimulus classes tested previously (8). Although Simas et al. (8) did not specify the equations used to generate their stimuli, we used the following equations to generate the gratings, based on their descriptions and examples:

Cartesian stimuli: $L(x, y)=L_{0}[1+\operatorname{csin}(2 \pi f u+\theta)]$

$$
u(x, y)=x \cos \phi-y \sin \phi
$$

angular stimuli: $L(x, y)=L_{0}[1+c \cos (2 \pi f a+\theta)]$

$$
a(x, y)=\tan ^{-1} \frac{\mathrm{y}}{\mathrm{x}}
$$

where $L_{0}$ is the mean luminance of the stimuli, $c$ is the contrast, $\theta$ is the phase, $f$ is the spatial frequency, and $\phi$ is the grating orientation. Frequencies were between 0.48 and 15.34 $\mathrm{c} / \mathrm{deg}$ for the Cartesian stimuli and between 2 and $32 \mathrm{c} / 360^{\circ}$ for the angular stimuli. Stimulus phases and orientations were the same as those used previously (8), i.e., vertical grating bars and angular gratings with maximum luminance set to the right.

The average luminance of the stimulus was $6.5 \mathrm{~cd} / \mathrm{m}^{2}$ and viewing was binocular from a distance of $105 \mathrm{~cm}$. We set the background luminance at $4.8 \mathrm{~cd} / \mathrm{m}^{2}$, since in the previous study (8) the background luminance was set at approximately $76 \%$ of the mean stimulus luminance. Environment illumination was around $0.5 \mathrm{~cd} / \mathrm{m}^{2}$.

\section{Procedure}

Thresholds were determined using a twoalternative forced-choice algorithm. The observers were asked to fix their eyes on a small mark made in the center of the screen. One of two successive exposures contained the target stimulus and the other stimulus exposure contained a zero-contrast stimulus having the same mean luminance. Both exposures were denoted by an auditory tone and had a total duration of $2 \mathrm{~s}$. A 1-s interstimulus interval contained a zero-contrast screen having the same luminance as the mean luminance of the screen. Subjects were asked to shift their eyes and attention to a dark-green panel located above the monitor at the end of the second interval and to indicate whether the target stimulus was present during the first or the second exposure by pressing one of two keys on the computer keyboard. Decision time was unlimited (usually 1-2 s). Auditory feedback was given for an incorrect response. A new trial, signaled by a different tone, started $2 \mathrm{~s}$ after the last response and the subject had to shift his eyes and attention to the screen again. The interval from the new-trial-tone and the first test interval was $1.5 \mathrm{~s}$, estimated to be sufficient for the subject to find the fixation mark and accommodate his eye. After three consecutive correct responses the contrast of the target stimulus was decreased by a factor of $0.1 \mathrm{log}$ units, and after each incorrect response the contrast was increased by the same factor. Sixteen reversals were obtained for each stimulus and a threshold estimate at 
the probability level of 0.79 (11) was calculated as the mean of the last 12 reversals.

\section{Results and Discussion}

\section{Experiment 1}

Figure 1 shows the CSFs to Cartesian and angular gratings for the two subjects and as measured by Simas et al. (8). The CSFs
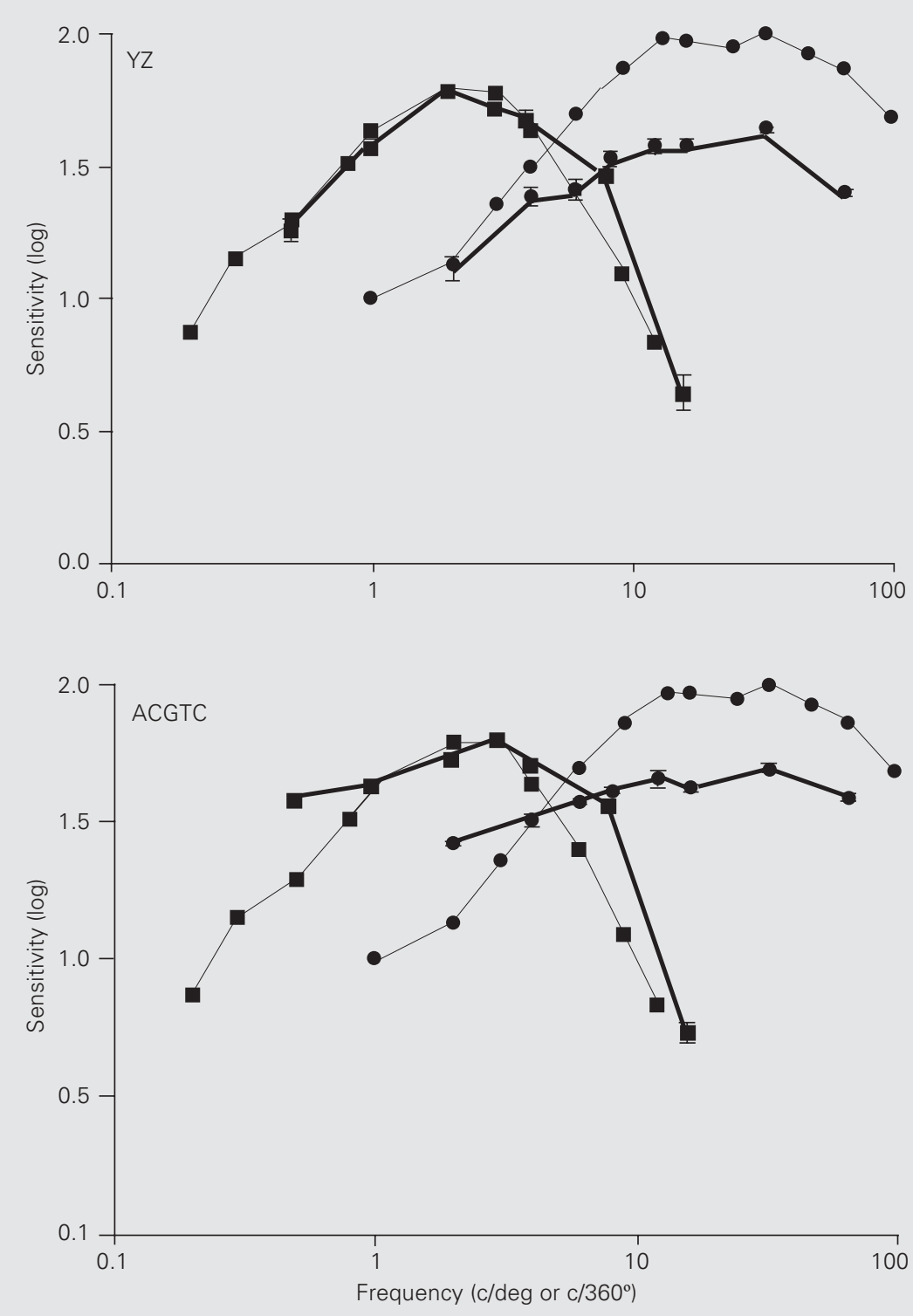

Figure 1. Contrast sensitivity for Cartesian (squares) and angular (circles) gratings as a function of spatial frequency (c/deg or $\mathrm{c} / 360^{\circ}$, respectively) from subjects $\mathrm{YZ}$ and ACGTC (thick lines; Experiment 1) and data from Simas et al. (8) (thin lines). Error bars indicate the SEM. measured for both subjects were similar and the CSFs peaked at approximately $3 \mathrm{c} / \mathrm{deg}$ and $32 \mathrm{c} / 360^{\circ}$ for the Cartesian and angular gratings, respectively. Their general form and peak sensitivity location were also similar to those registered in the previous study (8). However, the higher sensitivity to the angular stimuli reported previously was not confirmed: we found that peak sensitivity to angular gratings was on average $0.13 \mathrm{log}$ units lower than to Cartesian gratings for both subjects.

This major discrepancy could be a result of differences in experimental conditions and/or control level (Table 1). In the previous study (8) only 127 contrast levels were available and the voltage-luminance relation was not linearized. Bach and colleagues (12) estimated that under these conditions, in the luminance mid-range, contrast rose approximately $2.0 \%$ with each nominal step and not the presumed $0.8 \%$. In the current study this error was minimized by increasing the availability of luminance levels from 256 to 4096 , and consequently the number of contrast levels from 127 to 2047, for approximately the same luminance range. In addition, the 4096-luminance level look-up table was linearized up to $\mathrm{R}^{2}=0.9998$. Temporal and spatial aspects were also improved. Screen refresh rate was raised from $30 \mathrm{~Hz}$ interlaced to $85 \mathrm{~Hz}$ non-interlaced, guaranteeing a flicker-free image. Cartesian stimulus frequencies were chosen to match the monitor pixel size, which was decreased from 0.028 to 0.016 visual degrees, so as to reduce spatial aliasing. The total number of pixels was also doubled, which is especially significant for angular stimulus presentation, since luminance is positively correlated with the number of pixels per cycle (13). As angular stimuli contain very high frequency components, they are more susceptible to this physical artifact. In the current study we assured, as mentioned above, that each interval was observed for $2 \mathrm{~s}$, giving the subject $1.5 \mathrm{~s}$ to shift his fixation from the panel to the 
screen monitor and to accommodate his eyes. In the previous experiment, the time taken to change fixation was included in the first interval. Contrast steps were $0.1 \log$ units and threshold calculation was based on the average of the last 12 reversals out of 16 collected. In the previous study (8) one nominal contrast level was used for each contrast change and the threshold was the average of 20 collected reversals. Although the former procedure is faster, and thus easier for the observers, both result in a threshold estimate at the probability level of $0.79(11,14)$. Audio feedback in the present experiment was modified from positive to negative in order to reduce the general audio stimulation, although no significant alteration in the results was expected due to this difference.

There were a few minor differences in experimental conditions due to the limitations of our equipment. As a result of the use of the video attenuator, the stimuli were black-and-green luminance modulated, instead of black-and-white. This difference is not expected to significantly alter the results, since Van Nes (15) found no difference between the photopic CSFs for red, green or blue light modulation. This result was ob- tained after optical correction for chromatic aberration, but Mullen (16) measured the CSFs of black-and-green and black-and-yellow modulated stimuli and also found them very similar. A second difference in stimulus configuration between the two studies is the target/background area ratio, i.e., 0.16 as compared to the 0.23 ratio in the previous study. This small difference is not sufficient to explain such an opposite effect in results.

\section{Experiments 2 and 3}

In order to identify more conclusively the source of the discrepancy in the results, we repeated twice the experiment with subject YZ, this time with a degraded experimental control. In both experiments screen resolution was reduced from $1024 \times 768$ to $640 \mathrm{x}$ 480 pixels and the frame rate from 85 to 60 $\mathrm{Hz}$ non-interlaced. This is far from the $30 \mathrm{~Hz}$ interlaced frame rate in the previous study (8), but was the minimum available. The video attenuator circuit was used, but the number of available contrast levels was reduced from 2047 to 127 and no voltageluminance linearization was done. Stimulus generation and parameters were the same as

Table 1. Comparison of the equipment and conditions in Experiments 1, 2 and 3 and in the experiment conducted by Simas et al. (8).

\begin{tabular}{|c|c|c|c|c|}
\hline & Experiment 1 & Experiment 2 & Experiment 3 & Reference 8 \\
\hline Monitor resolution (pixels) & $1024 \times 768$ & $640 \times 480$ & $640 \times 480$ & $512 \times 480$ \\
\hline Screen refresh rate $(\mathrm{Hz})$ & 85 non-interlaced & 60 non-interlaced & 60 non-interlaced & 30 interlaced \\
\hline Contrast resolution (bit) & 12 & 8 & 8 & 8 \\
\hline LUT linearization & Yes & No & No & No \\
\hline Luminance modulation & Black-and-green & Black-and-green & Black-and-green & Black-and-white \\
\hline Reduced pixel aliasing & Yes & No & No & No \\
\hline Contrast step & 0.1 log units & 0.1 log units & 0.1 log units & 1 nominal unit \\
\hline Threshold calculation & 12 out of 16 reversals & 20 out of 20 reversals & 20 out of 20 reversals & 20 out of 20 reversals \\
\hline Stimulus/background ratio & 0.16 & 0.16 & 0.16 & 0.23 \\
\hline Audio feedback & Negative & Positive & Positive & Positive \\
\hline Time interval to shift fixation & Yes & No & No & No \\
\hline Fixation & Central & Central & Free & Central \\
\hline
\end{tabular}

LUT = look-up table 
in Experiment 1, the only exception being the frequencies of the Cartesian gratings, which were set at $1,2,3,4,8,16 \mathrm{c} / \mathrm{deg}$. These values were not matched to the pixel size (see Experiment 1), as in the previous study, and introduced some aliasing. No changes were made in the definition of angu-
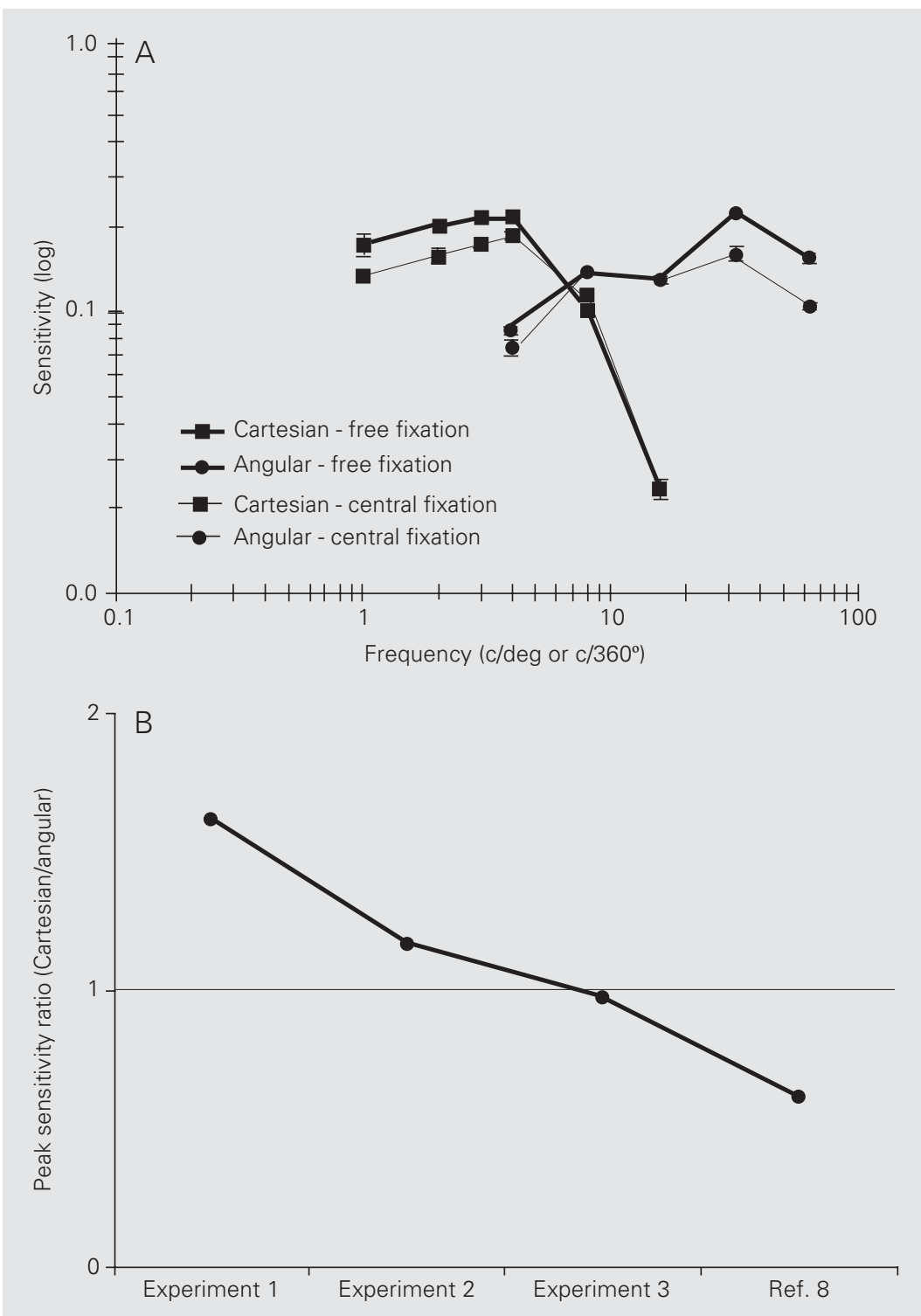

Figure 2. A, Contrast sensitivity for Cartesian (squares) and angular (circles) gratings as a function of spatial frequency (c/deg or $\mathrm{c} / 360^{\circ}$, respectively) from subject $\mathrm{YZ}$ at lower experimental control with central fixation (thick lines; Experiment 2) and free fixation (thin lines; Experiment 3). Error bars indicate the SEM. B, Ratios of the peak sensitivity to Cartesian and angular stimuli (Cartesian/angular) measured for subject $\mathrm{YZ}$ in Experiments 1, 2 and 3 , and by Simas et al. (8). The horizontal line is at the level where the peaks of the contrast sensitivity functions are equal. lar stimuli. For the calculation of the contrast threshold, we increased the number of reversals from 16 to 20 and the final threshold was the average of 20 reversals without any exclusion.

In Experiment 1 the subject was instructed to shift his eyes and attention from the dark panel to the screen at the beginning of a newtrial-tone. However, the 1.5-s prestimulus interval was eliminated and the stimulus appeared exactly at the beginning of the tone. The only difference between Experiments 2 and 3 was that in Experiment 2 the subject was instructed to fix his eyes on the dot in the center of the screen, like in Experiment 1 and in the previous study (8), and in Experiment 3 the subject was free to move his fixation anywhere in the area of the stimuli. Audio feedback was changed from negative to positive.

Both Experiments 2 and 3 were designed to partially reproduce the lower experimental control of the previous study (8). Experiment 3 was done in order to test the hypothesis that the different results found by Simas et al. (8) were due to differences in fixation of the stimuli by the subjects.

Figure 2A shows the results of Experiments 2 and 3. Each point represents the average of three measured thresholds. In both conditions, the Cartesian and angular CSFs had a shape and peak sensitivity location similar to those obtained in Experiment 1. However, the difference in peak sensitivity between the two stimuli was reduced from Experiment 1 to Experiment 2 and extinguished in Experiment 3. The ratios of the Cartesian and angular peak sensitivity, including that measured by Simas et al. (8) are plotted in Figure 2B. There is a clear shift from higher sensitivity to Cartesian stimuli to angular stimulus dominance. As the only difference between Experiments 1 and 2 was the control level over the experimental conditions, it seems that this factor is in part responsible for the opposite effect found by Simas et al. (8). The results of Experiment 3 indicate that the subjects studied by Simas et 
al. (8) may not have fixed their eyes on the dot in the center of the screen during the stimulus presentations.

The present study indicates that the peak sensitivity to angular gratings is $0.13 \mathrm{log}$ units lower than to Cartesian gratings. This result is in agreement with the most current models that assume Cartesian filtering at the first visual processing stage and that the CSF is determined by the highest amplitude Fourier component. Using as an example the stimuli that showed the highest sensitivity in each coordinate system, we analyzed the amplitudes of the Fourier components in the Cartesian $1.917 \mathrm{c} / \mathrm{deg}$ and angular $32 \mathrm{c} / 360^{\circ}$ stimuli. Figure 3 shows these stimuli (A) and their Fourier transformation (B and C). Most of the contrast energy of the Cartesian stimulus is concentrated in two points (in a single frequency at one orientation), while those of the angular stimulus are distributed radially and angularly (at different frequencies and orientations). Taking a radial section of the spectrum, we can see that the Cartesian stimulus peak amplitude is higher ( 0.22 log units) than that of the angular stimuli (Figure 3D), according to the contrast sensitivity difference found in Experiment 1.

However, current evidence does not exclude polar spatial analysis of suprathreshold stimuli by the human visual system. This idea received substantial psychophysical support in a study of the mechanisms underlying global form perception using Glass patterns (17). The stimuli consisted of a pattern of random dots that generated a percept of a global structure of radial, angular, hyperbolic and Cartesian patterns. Detection threshold was measured by the addition of

Figure 3. Fourier analysis of the Cartesian and angular stimuli with the highest sensitivity $(1.917 \mathrm{c} / \mathrm{deg}$ and 32 $\mathrm{c} / 360^{\circ}$, respectively). $A$, Stimuli that were used in Experiment 1 , and $B$ and $C$ their two-dimensional amplitude spectrum. $D$, Horizontal radial cross-section of the two-dimensional Cartesian and angular stimulus spectra (black and gray lines, respectively).
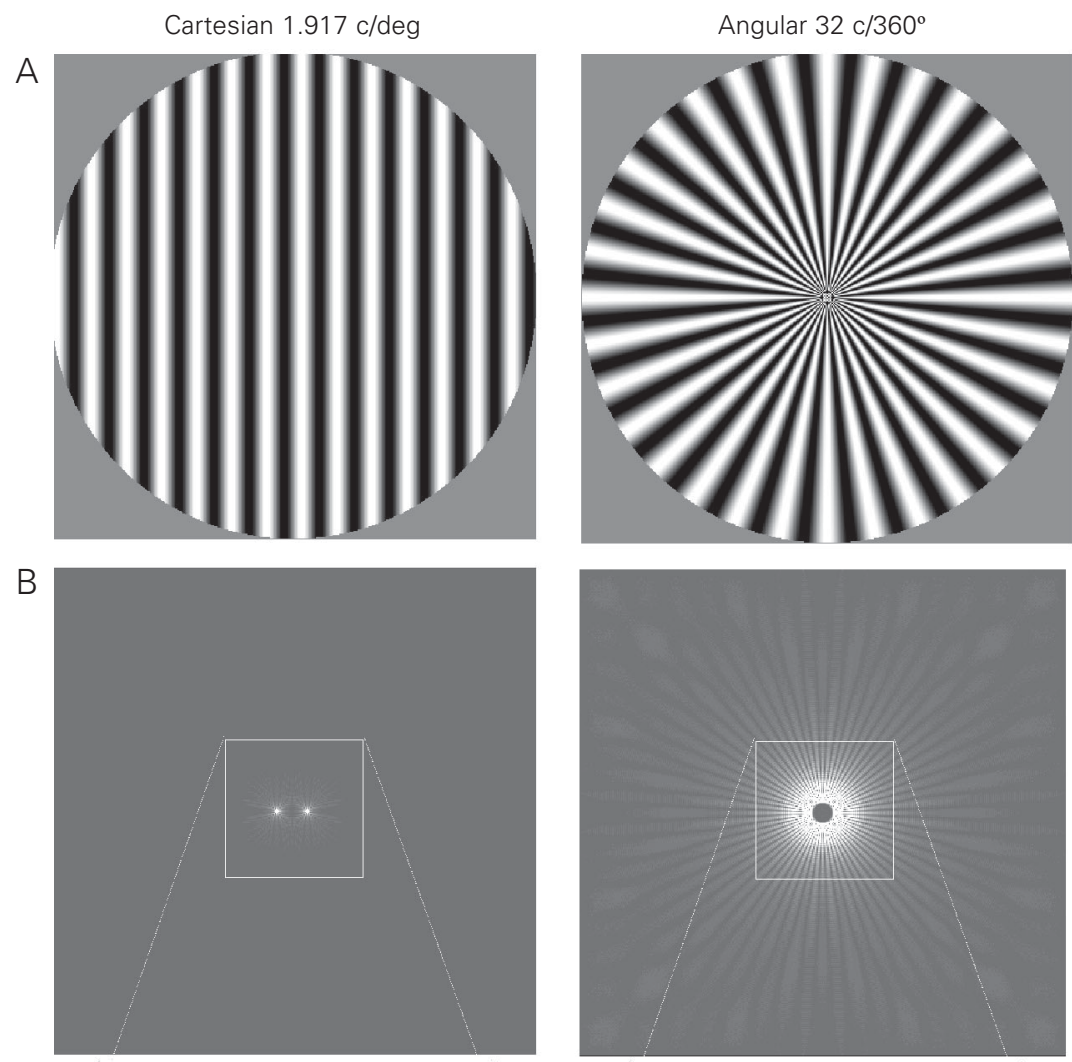

C
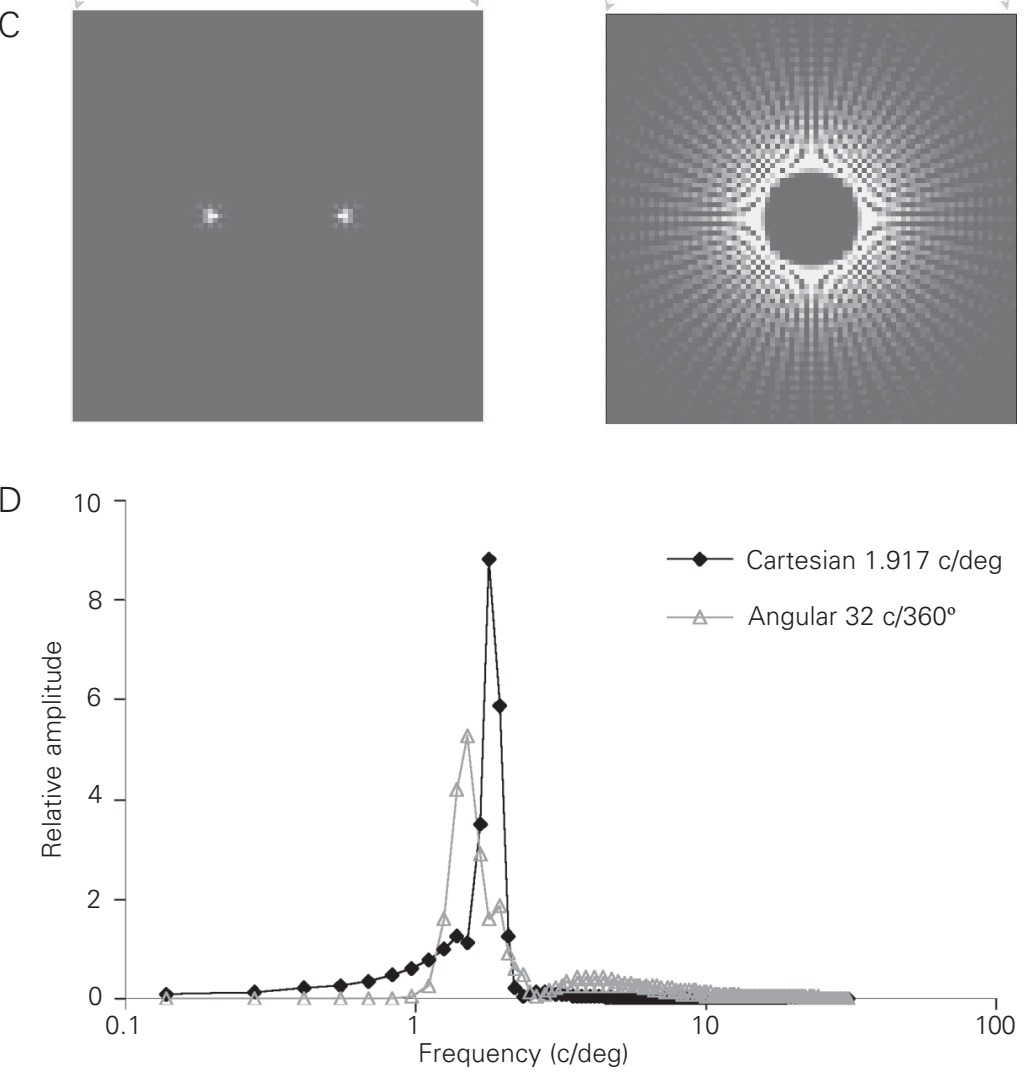
noise. Threshold decreased from Cartesian to hyperbolic, angular and radial patterns. Measurements of the thresholds as a function of the stimulated area showed global pooling (area estimated at 3.5-4.5 ${ }^{\circ}$ ) of orientation information in the detection of angular and radial Glass patterns, but only local pooling in the detection of Cartesian patterns.
These results also agree with several electrophysiological studies such as those by Gallant and colleagues $(18,19)$ and Mahon and DeValois (20), where it was shown that populations of cells in LGN, V1, V2 and V4 in monkeys have specific sensitivity to Cartesian, polar and hyperbolic stimuli.

\section{References}

1. Campbell FW \& Robson JG (1968). Application of Fourier analysis to the visibility of gratings. Journal of Physiology, 197: 551-566.

2. Robson JG (1975). Receptive fields: neural representation of the spatial and intensive attributes of the visual image. In: Carterette ED \& Friedman MP (Editors), Handbook of Perception. Vol. 5. Academic Press, New York.

3. Kelly DH (1960). Stimulus pattern for visual research. Journal of the Optical Society of America, 50: 1115-1116.

4. Kelly DH \& Magnuski HS (1975). Pattern detection and the twodimensional Fourier transform: circular targets. Vision Research, 15: 911-915.

5. Simas MLB \& Dodwell PC (1990). Angular frequency filtering: a basis for pattern decomposition. Spatial Vision, 5: 59-74.

6. Simas MLB, Frutuoso JT \& Vieira FM (1992). Inhibitory side bands in multiple angular frequency filters in the human visual system. Brazilian Journal of Medical and Biological Research, 25: 919-923.

7. Simas MLB \& Frutuoso JT (1997). Inhibitory effects in the detection of $1 \mathrm{cpd}$ Jo targets superimposed to angular frequency stimuli or sinusoidal gratings. Brazilian Journal of Medical and Biological Research, 30: 783-786.

8. Simas MLB, Santos NA \& Thiers FA (1997). Contrast sensitivity to angular frequency stimuli is higher than that for sinewave gratings in respective middle range. Brazilian Journal of Medical and Biological Research, 30: 633-636.

9. Simas MLB \& Santos NA (2002). Narrow-band 1, 2, 3, 4, 8, 16 and 24 cycles $/ 360^{\circ}$ angular frequency filters. Brazilian Journal of Medical and Biological Research, 35: 243-253.

10. Pelli DG \& Zhang $L$ (1991). Accurate control of contrast on microcomputer displays. Vision Research, 31: 1337-1350.
11. Wetherill GB \& Levitt H (1965). Sequential estimation of points on a psychometric function. British Journal of Mathematical and Statistical Psychology, 18: 1-10

12. Bach M, Meigen T \& Strasburger H (1997). Raster-scan cathode-ray tubes for vision research - limits of resolution in space, time and intensity, and some solutions. Spatial Vision, 10: 403-414.

13. García-Pérez MA \& Peli E (2001). Luminance artifacts of cathode-ray tube displays for vision research. Spatial Vision, 14: 201-215.

14. García-Pérez M (1998). Forced-choice staircases with fixed step sizes: asymptotic and small-sample properties. Vision Research, 38: 1861-1881.

15. Van Nes FL (1967). Spatial modulation transfer in the human eye Journal of the Optical Society of America, 57: 401-406.

16. Mullen KT (1985). The contrast sensitivity of human colour vision to red-green and blue-yellow chromatic gratings. Journal of Physiology, 359: 381-400.

17. Wilson HR \& Wilkinson F (1998). Detection of global structure in Glass patterns: implications for form vision. Vision Research, 38: 2933-2947.

18. Gallant JL, Braun J \& VanEssen DC (1993). Selectivity for polar hyperbolic, and Cartesian gratings in macaque visual cortex. Science, 259: 100-103.

19. Gallant JL, Connor CE, Rakshit S, Lewis JW \& VanEssen DC (1996). Neural responses to polar, hyperbolic, and Cartesian grating in area V4 of the macaque monkey. Journal of Neurophysiology, 76: 27182739.

20. Mahon LE \& DeValois RL (2001). Cartesian and non-Cartesian responses in LGN, V1, and V2 cells. Visual Neuroscience, 18: 973 981. 